\title{
Lidil
}

Revue de linguistique et de didactique des langues

$63 \mid 2021$

Littératie numérique et didactique des langues et des cultures

\section{Anda Fournel, Thea Ionescu et Jean-Pascal Simon (dir.), Geste, parole et conceptualisation, revue Studia Universitatis Babeş-Bolyai, Philisophia, vol. 64, $\mathrm{n}^{\circ} 2$ 2019, 212 p. <http://studia.ubbcluj.ro/download/pdf/1243.pdf>.}

\section{Aurélie Mariscalchi}

\section{OpenEdition}

\section{Journals}

Édition électronique

URL : http://journals.openedition.org/lidil/9113

DOI : 10.4000/lidil.9113

ISSN : 1960-6052

Éditeur

UGA Éditions/Université Grenoble Alpes

Édition imprimée

ISBN : 978-2-37747-283-3

ISSN : $1146-6480$

Référence électronique

Aurélie Mariscalchi, « Anda Fournel, Thea Ionescu et Jean-Pascal Simon (dir.), Geste, parole et conceptualisation, revue Studia Universitatis Babes-Bolyai, Philisophia, vol. 64, n² 2 », Lidil [En ligne], 63 | 2021, mis en ligne le 30 avril 2021, consulté le 30 avril 2021. URL : http://journals.openedition.org/ lidil/9113; DOI : https://doi.org/10.4000/lidil.9113

Ce document a été généré automatiquement le 30 avril 2021.

(C) Lidil 


\section{Anda Fournel, Thea Ionescu et Jean- Pascal Simon (dir.), Geste, parole et conceptualisation, revue Studia Universitatis Babeş-Bolyai, Philisophia, vol. $64, \mathrm{n}^{\circ} 2$}

2019, 212 p. <http://studia.ubbcluj.ro/download/pdf/1243.pdf>.

\section{Aurélie Mariscalchi}

\section{RÉFÉRENCE}

Anda Fournel, Thea Ionescu et Jean-Pascal Simon (dir.), Geste, parole et conceptualisation, revue Studia Universitatis Babeş-Bolyai, Philisophia, vol. 64, nº 2, 2019, 212 p. <http:// studia.ubbcluj.ro/download/pdf/1243.pdf>.

La revue Philosophia de l'Université roumaine Babeş-Bolyai publie en août 2019 son deuxième numéro de l'année. Fidèle à la ligne éditoriale de la revue, ce numéro, intitulé Geste, parole et conceptualisation sollicite le point de vue de chercheurs.euses en philosophie, sciences sociales et sciences du langage sur les liens qu'entretiennent le corps, le langage et la pensée dans le fonctionnement humain. Ce numéro fait suite au séminaire international «Gestes, paroles \& conceptualisation » (Université BabeşBolyai, Roumanie, 10-12 octobre 2018) qui a donné lieu à la publication de certaines des communications. Il rassemble ainsi six articles qui structurent leur réflexion à l'aune du concept d'embodiment (cognition incarnée) selon lequel « la connaissance dépend d'un monde inséparable de nos corps, de notre langage et de notre histoire culturelle - bref, de notre corporéité » (Varela et coll., 1993, p. 210), mais selon des perspectives différentes. 
2 L'article de Bulea-Bronckart propose une réflexion sur le rôle du langage dans le fonctionnement humain dans le cadre de l'interactionnisme socio-discursif. L'autrice explique que le développement humain se réalise grâce à un ensemble complexe d'interactions multiples qui se produisent dans un milieu particulier (c'est-à-dire un environnement physique et social) dans lequel la langue, imprégnée de valeurs sociohistoriques, a un rôle essentiel: faire émerger la pensée et structurer le psychisme. Dans l'article suivant, Bodea s'appuie sur la phénoménologie de Richir $(1988,2015)$ et propose une réflexion sur la construction du sens avec un intérêt particulier pour le langage. Il s'intéresse davantage au processus à l'œuvre dans cette construction qu'au résultat. Un processus cognitif bien sûr, mais aussi somatique : le langage ne se réalise pas seulement dans les mots, mais aussi dans les gestes et le regard de l'Autre qui permet de forger son identité, son existence.

3 L'article de Fournel et la contribution collective de Marian, Rogobete, Vescan, Ilie et Ionescu proposent une démarche de recherche-action pour aborder la thématique. Fournel s'intéresse aux émotions en tant que dimension corporelle dans l'élaboration de concept chez des enfants et des adolescents. Elle envisage l'émotion comme indicateur d'une pensée conceptuelle singulière en construction et met en avant le rôle fondamental de l'interaction dans la construction de cette pensée. Les autrices de l'article collectif se sont quant à elles intéressées au rôle des émotions et des éléments sensori-moteurs dans l'apprentissage de nouveaux mots chez de jeunes enfants. Leur étude montre qu'il est nécessaire de les impliquer corporellement dans leurs apprentissages, permettant ainsi un meilleur rappel en mémoire du vocabulaire. Elles concluent qu'il est impossible et de toute façon contre-productif de séparer les émotions, le corps et la cognition dans le cadre d'un apprentissage incarné (embodied learning).

4 Enfin, Apostol et Popa proposent des réflexions dans une perspective plus philosophique concernant la relation geste-parole-conceptualisation. Pour Apostol, le rapport à l'Autre, c'est-à-dire l'intersubjectivité, se construit dans le rapport corpschair qui s'opère, entre autres, par la caresse. En s'appuyant sur la philosophie de Henry (2000), l'autrice explique la distinction et la complémentarité entre le corps et la chair : le corps permet le mouvement (corps-objet, pré-sensoriel) et la chair permet de sentir (corps-sujet, siège des impressions sensorielles). La caresse permet alors de signifier à l'Autre son existence. Popa, quant à elle, envisage la danse comme alternative au toucher (p. 115). La danse serait comme un intermédiaire donnant à voir le corps de l'Autre et offrant aussi une vision de son propre corps. Elle considère la danse comme un art, mais aussi comme simple mouvement du corps, considérant que tous les corps vivants dansent lorsqu'ils rencontrent d'autres corps (p.112). Elle souligne les interactions permanentes qui s'exercent entre notre présence dans le monde et notre environnement, affirmant que plutôt que d'être un contenant, l'espace est une dimension de notre présence au monde, continuellement façonné et modifié par nos mouvements (p. 116).

5 Chacune des contributions rappelle la nécessité d'envisager l'être humain comme un tout indivisible pour mieux comprendre son fonctionnement. Elles mettent ainsi fin aux traditionnelles distinctions cœur/corps/esprit, et montrent l'interdépendance de ces trois entités ainsi que leurs constantes interactions, entre elles bien sûr, mais aussi avec leur environnement. Nous regretterons finalement de ne trouver que six articles dans ce numéro, majoritairement orientés dans une perspective philosophique et assez 
théorique. En effet, seuls deux articles proposent une réflexion orientée vers le terrain et montrent les bénéfices d'une telle conception de l'être humain. La problématique de l'embodiment est en effet très actuelle et a un impact considérable, notamment sur nos acquisitions et nos apprentissages. Des articles complémentaires dans ce sens auraient permis de mieux saisir les enjeux concrets liés à ce concept. Ce numéro offre néanmoins une réflexion pluridisciplinaire riche qui donnera sans aucun doute envie au lecteur d'approfondir la thématique de la cognition incarnée.

\section{AUTEURS}

AURÉLIE MARISCALCHI

LIDILEM (EA 609), Université Grenoble Alpes 